\title{
Workshop on Reliability Issues in Nanomaterials
}

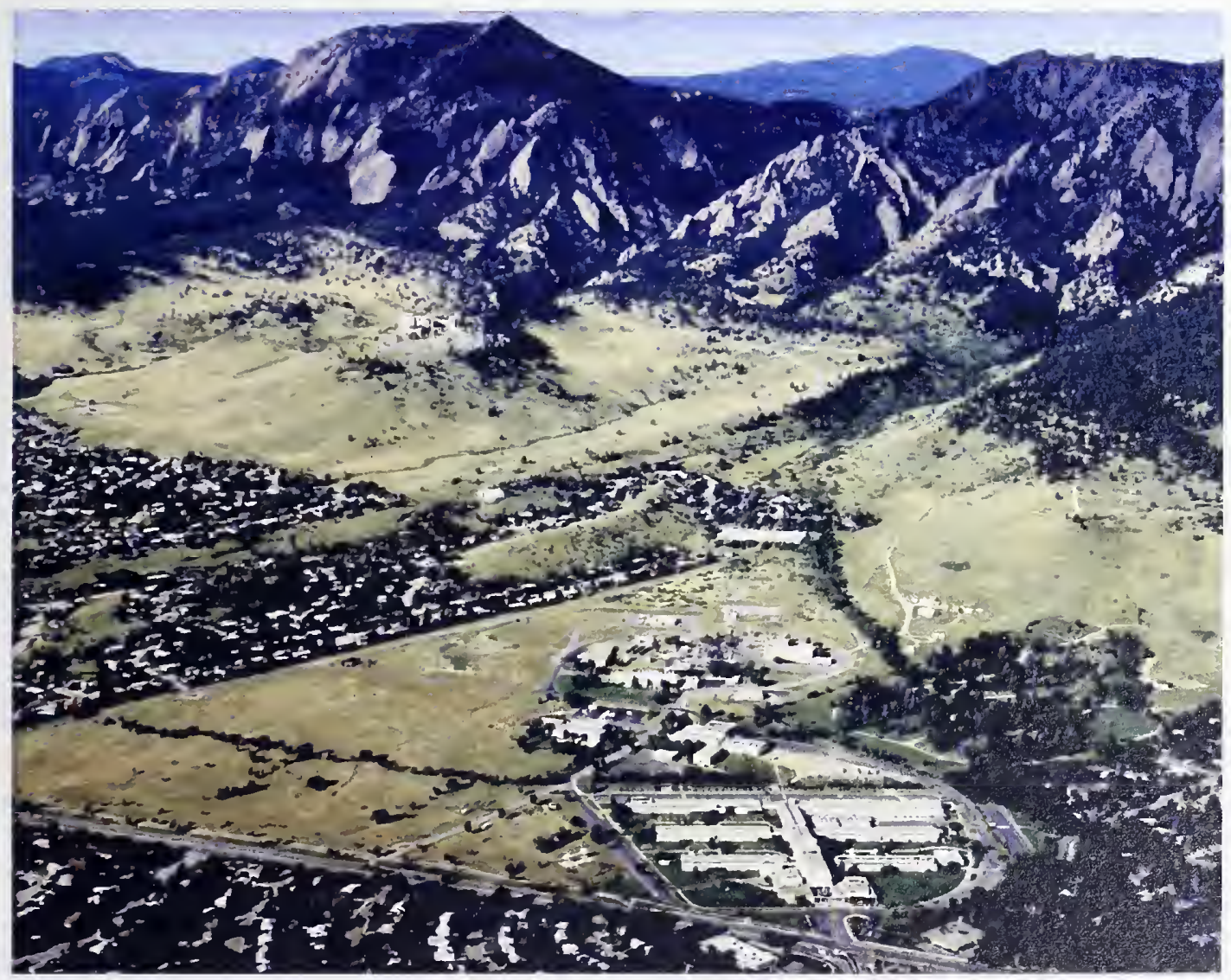

QC 100 ST National Instifute of Standards and Technology - Technology Administration - U.S. Department of Commerce 
he National Institute of Standards and Technology was established in 1988 by Congress to "assist industry in the dcvelopment of technology ... needed to improve product quality, to modernize manufacturing processes, to ensure product reliability ... and to facilitate rapid commercialization ... of products based on new scientific discoveries."

NIST, originally founded as the National Bureau of Standards in 1901, works to strengthen U.S. industry's competitiveness: advance science and engineering; and improve public health, safety, and the environment. One of the agency's basic functions is to develop, maintain. and retain custody of the national standards of measurement, and provide the means and methods for comparing standards used in science, engineering. manufacturing, commerce, industry, and education with the standards adopted or recognized by the Federal Government.

As an agency of the U.S. Commerce Department's Technology Administration, NIST conducts basic and applied research in the physical sciences and engineering, and develops measurement techniques, test methods, standards, and related services. The Institute does generic and precompetitive work on now and advanced technologies. NIST's research facilities are located at Gaithersburg, MD 20899, and at Boulder, CO 80303. Major technical opcrating units and their principal activities are listed below. For more information visit the NIST Website at http://ww. nist.gov, or contact the Publications and Program Inquirics Desk, 30 1-975-3058.

\section{Office of the Director}

-National Quality Program

-International and Academic Affairs

\section{Technology Services}

- Standards Services

- Teclmology Partnerships

- Measurement Services

- Information Services

-Weights and Measures

\section{Advanced Technology Program}

-Economic Assessment

-Information Teclmology and Applications

-Chemistry and Life Sciences

Electronics and Photonics Technology

\section{Manufacturing Extension Partnership \\ Program \\ Regional Programs \\ - National Programs \\ -Program Development}

\section{Electronics and Electrical Engineering}

\section{Laboratory}

-Microelectronics

Law Enforcement Standards

Electricity

Semiconductor Electronics

-Radio-Frequency Technology ${ }^{1}$

Electromagnetic Technology ${ }^{1}$

Optoelectronics ${ }^{1}$

-Magnetic Technology ${ }^{1}$

\section{Materials Science and Engineering Laboratory \\ -Intelligent Processing of Materials \\ - Ceramics \\ -Materials Reliability ${ }^{1}$ \\ -Polymers \\ - Metallurgy \\ - NIST Center for Neutron Research}

\section{Chemical Science and Technology}

\section{Laboratory}

Biotechnology

-Process Measurements

-Surface and Microanalysis Science

Physical and Chemical Properties ${ }^{2}$

-Analytical Chemistry

\section{Physics Laboratory}

-Electron and Optical Physics

-Atomic Physics

- Optical Technology

-Ionizing Radiation

-Time and Frequency ${ }^{1}$

'Quantum Physics ${ }^{1}$

\section{Manufacturing Engineering}

\section{Laboratory}

Precision Engineering

-Manufacturing Metrology

-Intelligent Systems

-Fabrication Technology

-Manufacturing Systems Integration

\section{Building and Fire Research Laboratory}

-Applied Economics

-Materials and Construction Research

Building Environment

Fire Research

\section{Information Technology Laboratory}

-Mathematical and Computational Sciences ${ }^{2}$

-Advanced Network Technologies

- Computer Security

-Information Access

-Convergent Information Systems

-Information Services and Computing

-Software Diagnostics and Conformance Testing

-Statistical Engineering 


\title{
NIST Special Publication 1043
}

\section{Workshop on Reliability Issues in Nanomaterials}

\author{
Robert R. Keller \\ David T. Read \\ Materials Reliability Division \\ Materials Science and Engineering Laboratory \\ Roop Mahajan \\ Department of Mechanical Engineering \\ University of Colorado
}

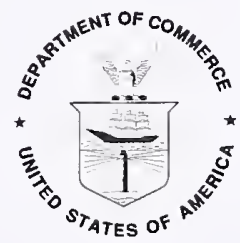

U.S. Department of Commerce Carlos M. Gutierrez, Secretany

Technology Administration Robert C. Cresanti, Under Secretary for Techmology 
Certain commercial entities, equipment, or materials may be identified in this document in order to describe an experimental procedure or concept adequately. Such identification is not intended to imply recommendation or endorsement by the National Institute of Standards and Technology, nor is it intended to imply that the entities, materials, or equipment are necessarily the best available for the purpose.

National Institute of Standards and Technology Special Publication 1043

Natl. Inst. Stand. Technol. Spec. Publ. 1043, 31 pages (January 2007)

CODEN: NSPUE2

For sale by the Superintendent of Documents, U.S. Government Printing Office Internet: bookstore.gpo.gov — Phone: (202) 512-1800 — Fax: (202) 512-2250

Mail: Stop SSOP, Washington, DC 20402-0001 


\section{CONTENTS}

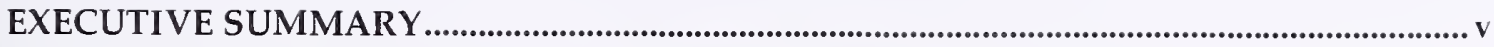

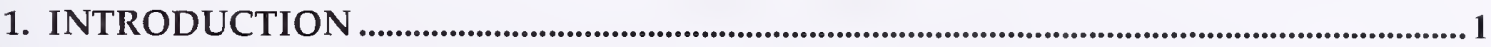

2. SCOPE

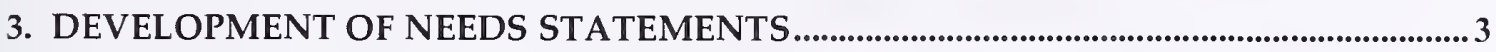

4. OUTLINE OF WORKSHOP REPORT............................................................................4

5. ASSESSMENT OF CURRENT KNOWLEDGE IN NANOMATERIALS FABRICATION AND CHARACTERIZATION

5.1 Reliability Issues Pertinent to Nanomaterials.......................................................................4

5.2 State of the Art in Measurement Technology for Reliability Studies of Nanomaterials ...........6

6. DETAILED SUMMARY OF NANOMATERIALS ISSUES ...............................................8

6.1 Nanomechanical challenges and needs in manufacturing and materials processing, nanomaterial products, advanced interconnects, and active devices ..........................................

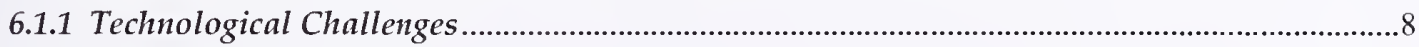

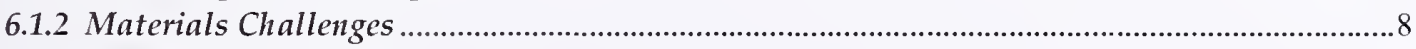

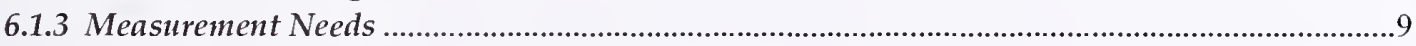

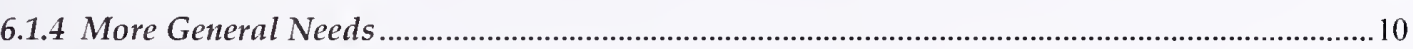

6.2 Nanomechanical challenges and needs in characterization and testing ...................................10

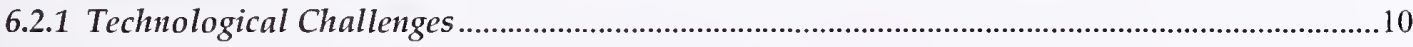

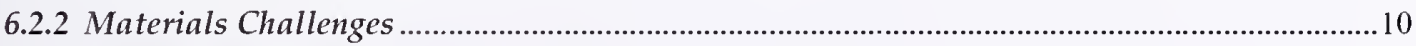

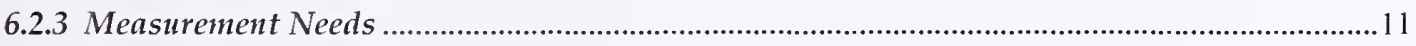

6.2.4 More General Needs ................................................................................................................

7. BRIEF SUMMARY OF ACTION ITEMS FOR METROLOGY DEVELOPMENT .............12

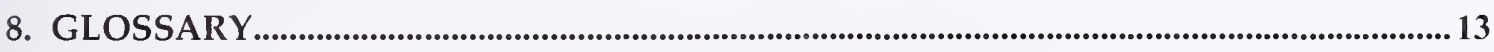

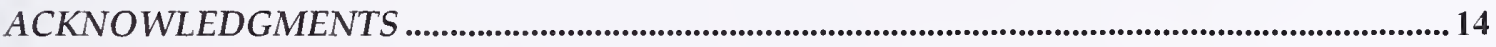

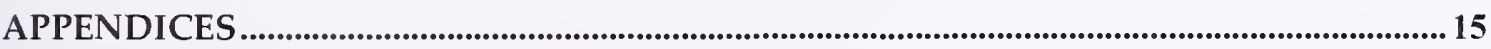

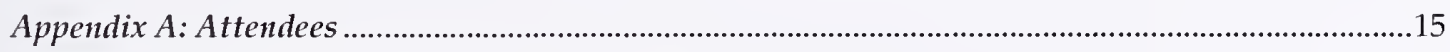

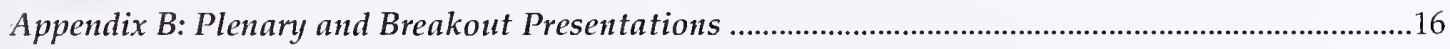

Appendix C: Workshop Program .....................................................................................................

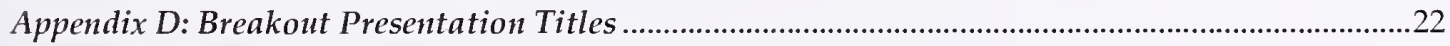

Appendix E: Questions Posed to Plenary Session Speakers...................................................................24

Appendix F: Questions Posed to Breakout Session Speakers..............................................................25

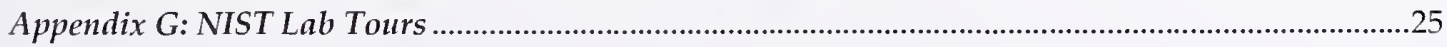




\title{
Workshop Organizers
}

Robert R. Keller

David T. Read

Materials Reliability Division, Materials Science and

Engineering Laboratory

National Institute of Standards and Technology

Roop Mahajan

Mechanical Engineering Department

University of Colorado

\author{
Venue \\ National Institute of Standards and Technology \\ Building 2, Room 0113 \\ U.S. Department of Commerce Laboratories \\ 325 Broadway \\ Boulder, Colorado 80305
}




\section{EXECUTIVE SUMMARY}

The Workshop on Reliability Issues in Nanomaterials was held at the Boulder Laboratories of the U. S. Department of Commerce on August 17-19, 2004. It was organized by the National Institute of Standards and Technology (NIST) and was designed to promote a particular subset of NIST's responsibilities under the National Nanotechnology Initiative (NNI). Larger issues, such as the definition, prospective uses, and impact of nanomaterials have been addressed elsewhere and were not considered in this workshop. The goal was to achieve consensus on two related topics: the identification of specific measurement-related barriers to successful incorporation of reliable nanomaterials into widespread engineering practice in the next 5 to 10 years; and the identification of measurement methodologies, standards, data, and models that might be appropriate for overcoming these barriers. 34 participants, representing cutting-edge nanomechanics-related research and development in industry, academia, and national laboratories, attended and contributed. Adding NIST-Boulder staff who attended brings the total to about 40 . The workshop format promoted discussion on the intended topics, and included

1. a pre-workshop questionnaire to be addressed by plenary and breakout speakers;

2. invitation of 10 plenary speakers who gave hour-long presentations and 23 breakout speakers, who gave brief presentations addressing the points raised in the questionnaire.

Key Conclusions: Industrial, academic, and national laboratory consensus indicated that there is always a need to understand fundamental causes of failure. Such understanding should then lead to redesign that is more reliable and to improved manufacturing. The goal of accurate performance and lifetime prediction for nanomaterials depends on the interplay between accurate materials testing and characterization, and reliability models incorporating valid measured data. Attendees agreed that while the synergy among industry, academia, and national laboratories was effective, more fundamental materials research is needed, where the actual division of labor would be determined by market forces and policy.

It was suggested that NIST could serve the unique role of developing metrology, standards, and materials characterization methods for improving reliability of nanomaterials. The most challenging and general metrology recommendation was the development of an "atom imager," a hypothetical instrument capable of nondestructively measuring the chemical identity and precise three-dimensional position of every atom within a nanomaterial. Such an instrument was postulated to be the key tool for optimizing fabrication/manufacturing and controlling reliability of nanomaterials. Nearer-term recommendations centered on improving the metrological performance of scanned probe microscopy (SPM) and nanoindentation. A secondary theme in many presentations was the need for modeling to be formally coupled with physical measurement in any study of nanomaterials; however, no novel computational tool or dataset was identified as a need of high priority. 
Acknowledgments: We are grateful for financial support from The National Science Foundation Solid Mechanics and Materials Engineering Program, the NIST Office of Microelectronics Programs, and the NIST Materials Reliability Division.

Robert R. Keller

David T. Read

Roop Mahajan

February 2005 


\title{
Workshop on Reliability Issues in Nanomaterials
}

\author{
Robert R. Keller and David T. Read \\ Materials Reliability Division \\ Materials Science and Engineering Laboratory \\ National Institute of Standards and Technology \\ Boulder, CO 80305 \\ Roop Mahajan \\ Department of Mechanical Engineering \\ University of Colorado \\ Boulder, CO 80305
}

The Workshop on Reliability Issues in Nanomaterials was held at the Boulder Laboratories of the U. S. Department of Commerce on August 17-19, 2004. It was organized by the National Institute of Standards and Technology (NIST) and was designed to promote a particular subset of NIST's responsibilities under the National Nanotechnology Initiative (NNI). Attendees agreed that while the synergy among industry, academia, and national laboratories was effective, more fundamental materials research is needed, where the actual division of labor would be determined by market forces and policy. It was suggested that NIST could serve the unique role of developing metrology, standards, and materials characterization methods for improving reliability of nanomaterials. The most challenging and general metrology recommendation was the development of an "atom imager," a hypothetical instrument capable of nondestructively measuring the chemical identity and precise three-dimensional position of every atom within a nanomaterial. Such an instrument was postulated to be the key tool for optimizing fabrication/manufacturing and controlling reliability of nanomaterials. Nearer-term recommendations centered on improving the metrological performance of scanned probe microscopy (SPM) and nanoindentation.

Keywords: design; experiment; fabrication; failure; lifetime prediction; material characterization; metrology; modeling; processing

\section{INTRODUCTION}

Nanomaterials lie at the heart of the field of nanotechnology. The NNI projects this field to have tremendous global impact, estimated to become a \$1 trillion industry over the next 15 to 20 years, with one third of that focused on materials and materials processing. At present, with an estimated \$1 billion annual investment, U.S. government spending has doubled in the last 4 
years and constitutes nearly one third of the world governments' annual investment of over $\$ 3$ billion. Through the NNI, approximately $22 \%$ of that U.S. investment addresses nanostructured materials by design, and another $39 \%$ addresses nano-electronics, -photonics, and -magnetics; the rest cover other NNI grand challenges such as health care, environment, energy, instrumentation and metrology, and manufacturing.

The introduction of nanomaterials into current and future technologies opens up an entirely new suite of both materials science and measurement science challenges. Effects of dimensional scaling play a stronger role in the reliability of nanomaterials than in any other materials known to date. Surfaces and interfaces can easily dominate and change behaviors and properties known to develop in bulk materials of the same chemical composition. As a result, one cannot simply extrapolate what is known about bulk material behavior to the nanoscale and expect to predict structure or properties accurately.

The full realization of reliable devices at this scale is limited by a host of materials science and engineering barriers centered on nanomechanics. For example, the development of accurate and repeatable metrologies for determining mechanical properties of materials is key to understanding how issues such as plasticity, fracture, adhesion, friction, stress/strain response, micro- and nanostructure, and chemistry determine the mechanical response of materials at the nanoscale. Further, nanomechanical response plays an important, yet sometimes indirect, role in other types of reliability, such as thermal or electrical reliability. Valid measurements can provide a foundation for building predictive models of nanostructure and behavior that will be based on materials science, as opposed to being purely empirical in nature.

It was the intent of this workshop to identify and discuss specific measurement-related barriers to successful incorporation of reliable nanomaterials into widespread engineering practice in the next 5 to 10 years, as determined by leading researchers in nanomechanics and reliability. The workshop also intended to identify measurement methodologies, standards, data, and models that might be appropriate for overcoming these barriers.

This report is also available online at http://www.boulder.nist.gov/div853/RIN .

\section{2. $S C O P E$}

These topics of discussion described the workshop for invitees considering attendance:

- Nanomaterials processing and fabrication and their effects on mechanical reliability

- Methods and techniques for characterizing structure of nanomaterials

- Methodologies for measuring mechanical properties in nanomaterials

- Experimental and modeling approaches to understanding effects of structure and dimensional scaling on mechanical behavior of nanomaterials, with emphasis on surface and interface issues

- Approaches to understanding what roles nanomechanics plays in other types of reliability

- Structure, property, and lifetime prediction 
Invitations to potential attendees informed them that we were seeking not only a description of the present state of the art in nanomechanical reliability, but also a glimpse into the future of the possible paths into which nanotechnology-based research could lead us in materials science and engineering, and vice versa.

This workshop addressed some of the Grand Challenges identified during the Nanomechanics Breakout Track of the NNI Interagency Workshop on Instrumentation and Metrology, held January 2004 at NIST-Gaithersburg. Namely, we addressed many of the following issues:

- Modeling of nanomechanical experiments;

- Integration of multiple techniques for measurement and characterization

- Experimentation/testing under real application conditions

- Standardization and calibration

- Instrument development for nanomechanics

- High-throughput, automated nanomechanics measurements

\section{DEVELOPMENT OF NEEDS STATEMENTS}

The last two hours of the workshop were used to review a two-part summary, written by the NIST organizers, of the measurement needs expressed by the attendees. One part was intended to be a complete list of specific needs expressed by all speakers, grouped according to possible metrological solutions. The other part was a shorter list of the consensus summary of the needs identified at the workshop. It became clear during the course of the workshop that materials researchers who are concerned with understanding and improving nanomechanical reliability commonly use several main tools:

- Nanoindentation

- Micro- and nanotensile and compression testing

- Scanned probe microscopy

- Modeling

A number of other important tools were discussed, for example, reflectivity measurement, for reflective coatings on mirrors intended for switching light signals to and from optical fibers, and scanned x-ray diffraction, for analysis of grain structure in copper on large silicon wafers; but the degree of convergence on nanoindentation, scanned probe microscopy, and modeling was neither intended nor anticipated in the planning of the workshop. As a result, most of the detailed and consensus need statements center around these measurement instruments.

The idea of the measurement issues important to researchers from different industries being addressed by a smaller suite of measurement tools was crystallized by one of the attendees in a breakout presentation. She said: "We care about different technologies and reliability concerns, but are addressing the same problems." 


\section{OUTLINE OF WORKSHOP REPORT}

We present an assessment of current nanomaterials fabrication and characterization knowledge in the context of reliability, based on questionnaires and discussion during the plenary sessions, and follow with a detailed summary of the issues of concern for making reliable nanomaterials, as articulated by all workshop attendees. Plenary speakers reviewed this report prior to its distribution.

Two alternative definitions of the reliability of nanomaterials are considered, as used in the context of this workshop:

1. the extent to which nanomaterials exhibit consistent mechanical behavior and associated properties over a long period, such as during a device lifetime;

2. the extent to which nanomaterial behavior and properties can be predicted over a given period.

The first definition is similar to the conventional use of the term "reliability." However, the second definition must sometimes be used in the design of devices and products, in the event that a material changes or degrades with increasing exposure to design stresses and environments. If the material degrades over time, but the degradation can be well predicted, the material may still be useful. In general, a "reliable" nanomaterial is one that can serve its purpose over the course of an intended device or designed product lifetime.

\section{ASSESSMENT OF CURRENT KNOWLEDGE IN NANOMATERIALS FABRICATION AND CHARACTERIZATION}

\subsection{Reliabillity Issues Pertinent to Nanomaterials}

Successful incorporation of nanomaterials into widespread engineering use requires us to understand why, how, and when failure will occur. Basic research addressing the combination of identification and measurement of failure mechanisms in these novel materials will provide the framework necessary to develop reliability models that are able to accurately predict changes in behavior and properties, and therefore lifetime, a key factor in successful manufacturing.

A number of factors that compromise reliability were identified during the workshop. The detailed lists as articulated by attendees follow in the next section, and are summarized here. We have sorted the reliability problems into three categories:

\section{Structures, behaviors, and resulting properties}

- Nanomaterials have much larger surface-to-volume ratios than bulk materials. This affects friction and wear properties as well as stiction in nanomaterials.

- Polycrystalline nanomaterials contain a higher fraction of grain boundary volume, and may be more susceptible to failure mechanisms involving such boundaries, including 
high-temperature deformation modes becoming operative at lower temperatures or failure mechanisms involving diffusion.

- Nanomaterials are almost always used as part of a materials system, and invariably come into contact with other materials, creating interfaces. Failure mechanisms involving delamination or chemical interdiffusion across an interface can be exacerbated. Diffusion through interfaces can also contribute to failures.

- Defect stability differs from that observed in bulk materials due to stronger influences of surfaces. Strain hardening may not follow the stages seen in bulk materials since dislocations can easily escape. Internal stresses associated with defects can build up, occupying the entire volume of a nanomaterial faster than might be expected from observations of bulk materials. Dislocations may not be energetically favored to exist in extremely small structures, forcing other mechanisms of plasticity or fracture to operate.

- Grain size is usually smaller in polycrystalline nanomaterials than in bulk materials, causing defect behavior and properties such as strength to differ from those seen in bulk materials.

- Localized behavior is much more important in nanomaterials. For example, an unusual orientation of a single grain can compromise the reliability of an electronic interconnect.

\section{Operation under extreme conditions}

- Accelerated tests must be developed in manners that suitably reproduce expected operating conditions, but sometimes the expected conditions can lead to behaviors not yet well understood in nanomaterials.

- Area- or volume-normalized external loads can be much more intense than in the case of bulk materials. Nanomaterials are often expected to withstand current densities, electric fields, pressures, stresses, or optical power densities that far exceed the values typically applied to bulk materials. Behaviors under such conditions are largely unknown.

- Time dependence of failure is very different from that observed in bulk materials, due to vastly different boundary conditions and length scales.

- Thermal management in nanomaterials is difficult, due to the high density of interfaces in multi-material systems. This is exacerbated by very high temperatures often undergone during thin- film or high-pressure processing.

\section{Size, shape, and distribution control}

- Ordered arrays or patterns of some nanostructures cannot presently be sufficiently fabricated. Quantum dots or carbon nanotubes must be made uniformly over large areas in order for many potential devices to be realized. Arrays and patterns must contain well controlled size and structure distributions. Lithographic approaches may not be 
feasible beyond certain dimensional limits, suggesting the need for self-assembly or other patterning processes.

- Stresses and strains can become very large in nanomaterials, and their management becomes extremely difficult. Although lattice-mismatched quantum dots (QDs) require high elastic strains ( $7 \%$ for InAs/GaAs) in order to exhibit their unique electronic behaviors, plastic relaxation must be avoided.

\subsection{State of the Art in Measurement Technology for Reliability Studies of Nanomaterials}

Reliability studies of nanomaterials depend on valid measurement of the properties and behaviors of interest. Thorough understanding of mechanical properties and behavior of nanomaterials requires accurate knowledge of the external forces and displacements applied to such materials, as well as the corresponding response of those materials.

Many test and characterization (i.e., measurement) methods were discussed during the workshop. Some are now in use, while others are still under development. In general, high performance tools for measuring bulk materials are commonly used for nanomaterials characterization, with appropriate modifications. For example, high resolution imaging methods such as atomic force microscopy (AFM), scanning tunneling microscopy (STM), transmission electron microscopy (TEM), and scanning electron microscopy (SEM) are routinely pushed to their limits. X-ray diffraction (XRD) is also used extensively for structural characterization of both aggregates and individual nanostructures. Focused ion beam (FIB) microscopy and manipulation has become a necessity for direct imaging, preparing specimens for other types of imaging, and patterning. Advances in mechanical testing and characterization have led to unprecedented resolutions in measurement and control of force and displacement. We summarize these state of the art methods here, categorized by application. A detailed listing of testing and characterization needs exceeding the present state of the art, as articulated by attendees, follows in the next section.

\section{MEMS/NEMS}

- Nondestructive and/or noninvasive measures for mechanical displacement, both static and dynamic, using electrical or optical methods

- Automated systems for testing at high throughput

- Surface analysis and analytical tools for identifying impurities or contaminants, e.g., Auger electron spectroscopy, X-ray photoelectron spectroscopy, secondary ion mass spectroscopy (SIMS)

- Environmental chambers for accelerated testing

- Shock and vibration testing at the product level

- Thermal shock and cycling tests for delamination and crack growth 


\section{Nanoscale Manufacturing and Assembly}

- STM methods for moving and removing atoms, to enable bottom-up fabrication

- Patterned atomic layer deposition (ALD) using STM for patterning (idea near realization)

- Surface acoustic wave (SAW) devices, accelerometers for mechanical properties

- Indirect optical methods using lasers, deflection techniques for displacement measurement

- Assembled micro-SEM columns to enable novel nanoscale characterization on a chip

- Assembled linear actuators to enable nanoscale testing on a chip

\section{Advanced Electronic Interconnect}

- Scanning XRD (four-circle goniometer) with area detector for simultaneous measurement and mapping of crystallographic phases, texture, and film thickness on $200 \mathrm{~mm}$ wafers; includes $20 \mu \mathrm{m}$ collimation. Development is underway to make this system apply to films of thickness $<10 \mathrm{~nm}$, where electrical methods are invalid. Such a system can be used in-line during manufacturing.

\section{Semiconductor Nanostmictures}

- Cleaving in situ within STM under ultra-high vacuum for cross-sectional imaging with atomic resolution

- Spectroscopy within STM for bandgap measurement as a function of position across a wafer

- Wafer curvature for average stress determination

- AFM-based patterned nanostructure fabrication using a nano-jet probe

- FIB nanopatterning for nanostructure position templates

\section{Mechanical testing}

- Young's modulus (E) and hardness (H) by continuous stiffness nanoindentation system and hybrid tribology/nanoindentation system; measurements still not in good agreement, with standard deviations as high as $\sim 75$ to $125 \mathrm{GPa}$ for E, $\sim 12$ to $14 \mathrm{GPa}$ for $\mathrm{H}$ for the case of thermal plasma chemical vapor-deposited (CVD) silicon carbide;

- Calibration of tip shape by use of fused quartz (not optimal)

- Nanoindentation measurements for $\mathrm{H}$ from sub-500 $\mathrm{nm}$ grains 
- Modified nanoindentation measurements of sub-40 nm Si nanoparticles

- Stressed overlayer and edge lift-off tests for adhesion;

- Nanoscale scratch tests for adhesion

- Acoustic AFM for mapping of elastic properties

\section{DETAILED SUMMARY OF NANOMATERIALS ISSUES}

The organization of this summary follows the session titles as listed in the program. These lists were reviewed in detail at the final session of the workshop, and are sorted by topical area as presented in the workshop. There is no significance to the order in which the issues are listed.

\subsection{Nanomechanical challenges and needs in manufacturing and materials processing, nanomaterial products, advanced interconnects, and active devices}

\subsubsection{Technological Challenges}

- Each device has its own unique reliability issues

- High yield and uniformity are required

- Reliability required over 20 year lifetimes

- Acceleration factor $<10$ desired in order to retain relevant physical mechanisms of reliability degradation; accelerating stresses include: temperature, voltage, current, humidity, strain, vibration, laser power

- Automated assembly manufacturing unavailable for parts of size $<0.5 \mathrm{~mm}$; all such assembly now done by humans

\subsubsection{Materials Challenges}

- MEMS and NEMS:

- Stiction (key issue)

- Possible solution: High-stiffness designs

- Friction and wear (sliding and impact)

- Possible solution: Noncontacting parts

- Hinge memory

- Fatigue and creep

- Possible solution: Conservative design stress

- Stress: need to absorb, balance, control

- Structure uniformity, defects in coatings for MEMS

- Mechanical integrity of polysilicon vs. metals

- Carbon Nanotubes (CNTs):

- Need dimensional uniformity, fewer defects

- Separate by electronic structure

- Can't make continuous fibers

- Need much better process control and qualification

- CNTs and Nanoparticles: 
- Separation, purification methods

- Nanocomposites:

- Need better understanding, using both simulation and experiments, especially $\mathrm{CNT} /$ matrix interface

- QDs:

- Need uniform size distribution and spacing

- Possible solution: Nanopatterning by FIB

- Control plasticity

- General issues:

- Make small things with more control over defects and uniformity of size

- Structure/property relationships for monolayers (adhesion, friction, wear)

- Effects of surface roughness

- Develop process variables $=f$ (residual stress, stress gradients, yield strength (YS), ultimate strength (UTS))

- Need to know where all the atoms are located

- Bulk property data on more materials, as foundation for nanomaterials:

- Elastic, dielectric, density of states

- Gradient characterization (chemical, physical), in complex matrices

\subsubsection{Measurement Needs}

- Tools and/or techniques to measure nanometer motion, addressing six degrees-offreedom, faster than device resonance frequencies

- Possible solution: optical surface profilometer, Doppler laser interferometer, strobing methods

- Tools for atomically precise engineering

- Possible solution: Patterned ALD, using STM

- Conversion of inherently two-dimensional measurement methods to three-dimensional methods

- Metrology for micro- and nanostructure of low-level features, especially early in the process

- Tools capable of multiple, coupled measurements with a single technique

- Thickness, crystalline phase, texture

- These must be done quickly

- Possible solution: scanning XRD

- Tools or systems capable of coupled measurements of properties and behaviors, using multiple techniques:

- In- situ, coupled measurements

- Optical coupled to mechanical methods

- Electrical coupled to mechanical methods

- High resolution (in space and energy), fast methods for imaging, morphology, chemistry

- Possible solution: improvements to SEM, AFM, TEM

- Methods for local measurement of: 
- Strain

- Electrical properties

- Structure size and/or shape

- Temperature, including spatially resolved, non-contact

- Possible solution: micro-Raman

- Inline-capable techniques for measurements during CMP:

- Particle size, morphology, dispersion, aggregation, correlation to performance

- High-throughput adhesion measurement

- New tests and/or models for new material combinations

- Nondestructive measurement of defects

- Deconvolution of sample-probe interaction in nanoindentation and scanned probe microscopy (SPM)

- Standards for:

- Reflectivity

- film thickness and texture

\subsubsection{More General Needs}

- Development of relationships between in situ tests and real failures

- More modeling coupled to experimental measurements

- Large-area temperature control during fabrication to promote structure uniformity

- More collaborations to stimulate development of relevant measurement needs:

- Manufacturing development and materials characterization/metrology development

- High-temperature interfacial reliability studies:

- Segregation, voiding

\subsection{Nanomechanical challenges and needs in characterization and testing}

\subsubsection{Technological Challenges}

- Small length scales:

- Difficult measurement problems even with state of the art instruments

- Need more than incremental advances in capabilities for mechanical behavior, beyond modification of existing tests, i.e., revolutionary, not just evolutionary

\subsubsection{Materials Challenges}

- In general (from a session announced for the Fall 2004 Materials Research Society Meeting), the dominant nanomaterial research problems at present include:

- Size effects, temperature or viscoelasticity, multiscale modeling, adhesion/surface energy

- Need good, repeatable test samples:

- No dislocations, surface defects 
- Rapid, fully automated fabrication methods suitable for production- and researchquality specimens

- Identification of specific problems and experimental approaches applicable to nanoscale biological materials

\subsubsection{Measurement Needs}

- Scaling of mechanical test methods to sub micrometer samples:

- Uniaxial, multiaxial, torsional stressing

- Fracture toughness

- Deformation mapping

- Gripping, manipulation

- Localized strain measurement

- Measurement of stress gradients, especially over small dimensions

- Valid methods for separating stress and temperature effects on stress-strain behavior

- Sub micrometer temperature measurement (direct)

- Improvements in spatially resolved characterization:

- SEM, SIMS, in-situ TEM, microdiffraction, micro-Raman, FIB (SE imaging)

- Single-atom resolution probes such as field ion microscopes

- "Desperately needed":

- High-resolution, in situ deformation studies in electron microscopes

- Nanoindentation:

- Instrument consistency for E, H

- Control of nanoindenter tip geometry

- Microforce standards

- Calibration standards for nano-test equipment

- Direct imaging of contact areas

- Improvements in position $(x, y, z)$ control and measurement

- AFM:

- Ability to do simultaneous mechanical testing and imaging

- Ability to do simultaneous nanodeposition and imaging

- Possible solution: dual-head AFM

- Tip/sample interaction energies need to be understood:

- Contact area effects

- Direct imaging of contact areas

- Need better characterization of:

- AFM tips

- Need sharp, durable, reproducible tips

- Need AFM and model velocities to match

- "Accurate and proven AFM technology!"

- Better control of SPM tip motion, i.e., no unintentional lateral motion

- Calibration standards for nano-test equipment

- Improvements in position $(x, y, z)$ control and measurement 
- The "perfect" AFM:

- Accurate knowledge of F and d for AFM

- F sensor perpendicular to surface

- Sharp, durable tip

- F sensor with stiffness control for both mechanical properties and topography

- Easy switch between F and d control

- Wide range of operational frequencies, temperature

- High-bandwidth, low-noise pre-amp

- Standards for:

- Microforce, both axial and lateral

- AFM spring constant

- AFM tip-specimen contact area

- Test/calibration specimens for nanoindentation and AFM-based mechanical testing

- Measurement of toughness and strength of thin films

- Standard bulk property data, for which accepted measurement methods exist, should be available for all relevant compositions and processes. (Needed as a basis for understanding properties of nanomaterials):

- Thin film and nanomaterial data should be disseminated as they become available

\subsubsection{More General Needs}

- MUST combine experiment and modeling for mechanical testing and adhesion/interfacial fracture.

\section{BRIEF SUMMARY OF ACTION ITEMS FOR METROLOGY DEVELOPMENT}

The lists of needs given above seem formidable at first glance. However, it became clear during the course of the workshop that there are several main tools commonly used by materials researchers who are concerned with understanding and improving nanomechanical reliability:

- Nanoindentation

- Micro- and nanotensile and compression testing

- Scanned probe microscopy

- Modeling

This short list of tools in no way diminishes the significance of others discussed during the workshop, e.g., electron microscopy and X-ray diffraction; it simply reflects a larger consensus about commonly used methods for nanomaterials. Attempts have been made to adapt these tools to most of the measurements listed, but considerable additional research and development are needed in order to meet the needs sufficiently. These "common-denominator" tools lead to the following action items for metrology development for nanomaterials reliability: 
Near term: Improved metrological performance of scanned probe microscopy and nanoindentation, for more quantitative measurements of position, displacement, force, temperature, and other relevant quantities;

Longer term: The "atom imager," a hypothetical instrument capable of measuring nondestructively the chemical identity and precise three-dimensional position of every atom in a nanomaterial. Newer generation field ion microscopes capable of imaging with single-atom resolution are under development, but are still subject to limitations such as difficult-to-control tip (which also serves as the specimen) structure and loss of atoms upon imaging (30 to $40 \%$ losses are not uncommon). Such instruments are also capable of sub-nanometer chemical spectroscopy.

\section{GLOSSARY}

AFM

ALD

CMP

CNT

CVD

d

E

F

FIB

G

$\mathrm{H}$

M

MD

MEMS

NEMS

NIST

QD

SAW

SE

SEM

SIMS

SPM

STM

TEM

UTS

XRD

YS
Atomic force microscope, atomic force microscopy

Atomic layer deposition

Chemical mechanical polishing

Carbon nanotube

Chemical vapor deposition

Displacement

Young's modulus

Force

Focused ion beam

Strain energy release rate

Hardness

Modulus

Molecular dynamics

Micro-electromechanical system

Nano-electromechanical system

National Institute of Standards and Technology

Quantum dot

Surface acoustic wave

Secondary electron

Scanning electron microscope, scanning electron microscopy

Secondary ion mass spectroscopy

Scanned probe microscopy

Scanning tunneling microscope, scanning tunneling microscopy

Transmission electron microscope, transmission electron microscopy

Ultimate tensile strength

$\mathrm{X}$-ray diffraction

Yield strength 


\section{ACKNOWLEDGMENTS}

The purpose of this Workshop was to gather and summarize the opinions of the attendees regarding the important and timely issue of measurement needs for Reliability Issues in Nanomaterials. The organizers gratefully acknowledge the preparation; travel time; attentive, constructive, and cooperative participation; and informed opinions contributed by the attendees. Special thanks are expressed to the majority of the attendees who stayed for the summary and review of the measurement needs at the end of the workshop, as well as to those who reviewed this final report. The organizers are grateful for critical reviews of the final report, provided by the plenary speakers.

This workshop was supported financially by The National Science Foundation Mechanics and Structure of Materials Program through Program Director Ken Chong, the NIST Office of Microelectronics Programs through director Steven Knight, and the NIST Materials Reliability Division. The bulk of the expenses were cost-sharing for the travel expenses of the attendees.

Wendy McBride, NIST Boulder Conference Program Manager, carried out the bulk of the arrangements for this workshop. The workshop benefited substantially from the well-organized meeting room, refreshments, and al fresco lunches that Wendy arranged. 


\title{
APPENDICES
}

The appendices to this report are intended to provide a historical record of the workshop to the extent practical. Most speakers have provided their presentations, mostly exactly as given at the workshop, some with confidential information removed. The discussions were not recorded.

\author{
Appendix A: Attendees \\ Arney, Susanne \\ Boyce, Brad \\ Burnham, Nancy \\ Chandross, Michael \\ Chong, Ken \\ Datta, Subhendu \\ de Boer, Maarten \\ Dunn, Marty \\ Gerberich, William \\ Goldman, Rachel \\ Greenberg, Alan \\ Holl, Susan \\ Hsu, Stephen \\ Hurley, Donna \\ Johnson, Harley \\ Keller, Robert \\ Lucent Technologies Bell Labs and NJNC \\ Sandia National Laboratories \\ Worcester Polytechnic Institute \\ Sandia National Laboratories \\ National Science Foundation \\ University of Colorado \\ Sandia National Laboratories \\ University of Colorado \\ University of Minnesota \\ University of Michigan \\ University of Colorado \\ Intel Corporation \\ NIST \\ NIST \\ University of Illinois at Urbana-Champaign \\ NIST \\ Kopycinska-Mueller, Malgorzata NIST \\ Mahajan, Roop \\ Moody, Neville \\ Pang, Mengzhi \\ Qi, H. Jerry \\ University of Colorado \\ Sandia National Laboratories \\ Cornell University \\ University of Colorado \\ Randall, John \\ Read, David \\ Zyvex Corporation \\ NIST \\ Reimanis, Ivar \\ Colorado School of Mines \\ Rodbell, Ken \\ Roshko, Alexana \\ IBM Corporation \\ NIST \\ Sbaizero, Orfeo \\ Schwaiger, Ruth \\ Sitaraman, Suresh \\ Smith, Douglas \\ University of Trieste (Italy) \\ Forschungszentrum Karlsruhe -IMF2 \\ Georgia Institute of Technology \\ NIST \\ Sokolov, Igor \\ Tewary, Vinod \\ Clarkson University \\ NIST \\ Uchic, Michael \\ Air Force Research Laboratories \\ Volinsky, Alex \\ University of South Florida \\ Yang, Bo \\ Zhu, Yong \\ Florida Institute of Technology \\ Northwestern University
}


Appendix B: Plenary and Breakout Presentations

Presentations from a majority of the speakers, both plenary and breakout, are included on the attached $C D$. For a listing of titles, please refer to appendices $C$ and D. 
Appendix C: Workshop Program

\section{Program, Day 1}

Tuesday, Augusst 17, 2004

8:00 a.m. Registration (coffee available)

8:30 a.m. Leslie Smith, Director, Materials Science and Engineering Laboratory, NIST Welcome

Topical Area: Fabrication

\section{Fabrication I: Nanomechanical Issues in Manufacturing Session Chair: David Read, NIST}

8:45 a.m. Susanne Arney, Lucent Technologies - Materials Requirements and Measurements in Design and Manufacture of Micro- and NanoElectromechanical Systems for Performance and Reliability

9:45 a.m. Break - coffee

10:00 a.m. John Randall, Zyvex - Materials Requirements and Measurements in Nanoscale Manufacturing and Assembly

11:00 a.m. Breakout 1:

Near-Term M/NEMS and Other Nanomaterial Products: Tools, Reliability, and Metrology

Chair: Dudley Finch, NIST/University of Colorado

12:30 p.m. Lunch (box lunch supplied)

1:30 - 2:00 p.m. $\quad$ Lab tours - Select ONE Tour

Tour 1. World Trade Center Investigation

Tour 2. Nanoscale Elastic-Property Imaging

Tour 3. Thin Film Mechanical Testing

Tour 4. MEMS for Testing of Biological Systems 


\section{Fabrication II: Nanomechanical Issues in Materials Processing Session Chair: Robert Keller, NIST}

2:00 p.m. Ken Rodbell, IBM - Materials Requirements and Measurements in Design and Manufacture of Advanced Electronic Interconnect for Performance and Reliability

3:00 p.m. Break

3:15 p.m. Rachel Goldman, University of Michigan - Materials Requirements and Measurements in Design and Fabrication of Semiconductor Nanostructures for Performance and Reliability

4:15 p.m. $\quad$ Breakout 2:

Advanced Interconnects and Active Devices: Reliability and Metrology Chair: Alexana Roshko, NIST

5:45 p.m. Adjourn for the day 


\section{Program, Day 2}

Wednesday, August 18, 2004

8:15 a.m. Ken Chong, National Science Foundation - NSF perspective on metrology development for nanomaterials

\section{Topical area: Characterization and Testing}

\section{Characterization and Testing I: Mechanics of Nanomaterials} Session Chair: Roop Mahajan, University of Colorado

8:30 a.m. William Gerberich, University of Minnesota - Significance of Mechanical Behavior in Small Volumes to Design of Reliable Nanomaterials and Nanoscale Devices

9:30 a.m. Break

9.45 a.m. Martin Dunn, University of Colorado - Necessary Reinterpretations of Continuum Mechanics Concepts for Applicability to Nanomaterials

10:45 a.m. Breakout 3:

Unusual Mechanical Properties of Nanomaterials Chair: Martin Dunn, University of Colorado

12:15 p.m. Lunch (box lunch supplied)

1:15-1:45 p.m. $\quad$ Lab tours - Select ONE Tour

Tour 1. World Trade Center Investigation

Tour 2. Nanoscale Elastic-Property Imaging

Tour 3. Thin Film Mechanical Testing

Tour 4. MEMS for Testing of Biological Systems 
Characterization and Testing II: Mechanical Probing Methods for Characterizing Nanomaterials Session Chair: Donna Hurley, NIST

1:45 p.m. Nancy Burnham, Worcester Polytechnic Institute - Role of Atomic Force Microscopy in Conception, Design, and Characterization of High-Performance and Reliable Nanomaterials

2:45 p.m. Break

3:00 p.m. Neville Moody, Sandia National Laboratories - Significance of Adhesion and Interfacial Fracture in Design of Reliable Nanomaterials and Nanoscale Devices

4:00 p.m. $\quad$ Breakout 4:

Atomic Force Microscopy and Nanoindentation

Chair: Ken Gall, University of Colorado

5:30 p.m. Adjourn technical sessions for day 2

5:45 p.m. Board bus for travel to Flagstaff Summit

6:00 p.m. Picnic, Flagstaff Stone Shelter (casual clothing suggested) 


\section{Program, Day 3}

Thursday, August 19, 2004

\section{Topical area: Nanomaterials metrology roadmap}

Session Chair: Stephen Hsu, NIST

8:30 a.m. Stephen Hsu, National Institute of Standards and Technology - The Nanomechanics Measurement Facility in the NIST Advanced Measurement Laboratory (AML)

8:45 a.m. Douglas Smith, National Institute of Standards and Technology Nanomechanics at NIST

9:45 a.m. Break

10:00 a.m. Robert Keller, National Institute of Standards and Technology - Summary of nanomaterials reliability issues and measurement needs

11:00 a.m. David Read, National Institute of Standards and Technology - Challenges, barriers, and priorities for nanomaterials metrology

12:00 noon Adjournment of the technical sessions of the workshop

1:00 p.m. Lab tours at University of Colorado, by request 1:00-5:00 p.m.

Report writing by volunteers 


\section{Appendix D: Breakout Presentation Titles}

Breakout 1: Near-Term M/NEMS and Other Nanomaterial Products: Tools, Reliability, and Metrology

Chair: Dudley Finch

DeBoer, Maarten In situ Property Measurement in MEMS

Greenberg, Alan Mechanical and Gas Transport Behavior of Dense Polymer Films

Sbaizero, Orfeo The Center for Excellence in Nanotechnology at the University of Trieste

Volinsky, Alex Thin Film Mechanical Reliability: Environmental Effects

Breakout 2: Advanced Interconnects and Active Devices: Reliability and Metrology

Chair: Alexana Roshko

Sitaraman, Suresh Off-Chip Interconnects: Design, Fabrication, and Reliability

Johnson, Harley Mechanical and Electronic Coupling in Nanotubes and Quantum Dots

Yang, Bo Energy Release Rate of Quantum Islands in Stranski-Krastanow Growth

Holl, Susan CMOS to beyond CMOS, Novel Material Characterization Challenges

Pang, Mengzhi Adhesion Measurements of Thin Film Structures 
Breakout 3: Unusual Mechanical Properties of Nanomaterials

Chair: Martin Dunn

\begin{abstract}
Uchic, Michael Mechanical Testing at the Micron-Size Scale
Schwaiger, Ruth Investigations of Nanostructured Materials - from Nanocrystalline to Nanoporous

Datta, Subhendu Measurement and Modeling of Thermomechanical Properties of Thin Layers

Qi, H. Jerry

Biomolecule Unfolding and Mechanics of Nacre

Reimanis, Ivar

Toughness Measurement of Thin Films: What Does it Tell Us and How Reliable is That?

Boyce, Brad

Measurement Challenges in Microtensile and Microfatigue Reliability Studies
\end{abstract}

Breakout 4: Atomic Force Microscopy and Nanoindentation

\title{
Chair: Ken Gall
}

Sokolov, Igor The Need in a Standard for Small Force to Probe Interactions in Nanoscale with the AFM

Zhu, Yong Novel Experiments to Assess the Mechanical Properties of Thin Films and Nano-Scale Structures

Chandross, Michael Simulations of Nanotribology 


\section{Appendix E: Questions Posed to Plenary Session Speakers}

Broad questions for all Plenary speakers; treat in terms of your area of expertise:

- What is considered state-of-the-art, in terms of experiment/processing/characterization and/or theory/modeling? (This may include some of your own work.)

- What are the problems now under investigation?

- What barriers (scientific and/or technological) do you foresee to advances in the coming 5 to 10 years? Please include barriers that may be addressed by improvements in materials measurements.

- What are possible approaches to solution? Please include how you might envision "division of labor," i.e., roles of industry, DOE labs, NIST, universities.

Questions for Fabrication speakers (Day 1, Arney, Randall, Rodbell, Goldman):

- How is mechanics/mechanical behavior important in terms of performance and reliability required at product level?

- How do you design-in performance and reliability, using principles of micro- and nanostructure control? Please comment on adequacy of existing lifetime or performance prediction methods.

- How does industry measure performance of both the end product and the constituent materials?

- What are the limitations/barriers of the present measures/metrology?

Questions for Characterization and Testing speakers (Day 2, Gerberich, Dunn, Burnham, Moody):

- Measurements of which properties do you foresee to be critical for advancing implementation of nanomaterials in the coming decade?

- How sufficient are the existing test methods for nanoscale property measurement? Can advances be made incrementally, or are revolutionary changes needed?

- Please comment on the role of modeling in your work. What answers do you seek? What type of modeling do you perform or refer to?

- What kind of information about materials structure is needed to guide the development of property characterization and testing methods? 


\section{Appendix F: Questions Posed to Breakout Session Speakers}

1. How does metrology affect your present work in nanomaterials?

2. What questions relating to reliability of nanomaterials would you like to see addressed in this workshop and report/roadmap?

3. What metrology issues are of the most significance to you for your work in the coming 5 to 10 years?

\section{Appendix G: NIST Lab Tours}

Tour 1. World Trade Center investigation - David McColskey In August of 2002, NIST took responsibility for the investigation of the World Trade Center disaster. The investigation objectives include determining why the buildings collapsed; the procedures and practices used in the design, construction, operation, and maintenance of the buildings; and areas in codes and practices that warrant revision. The eight interdependent projects include Project 3, "Analysis of Structural Steel," led by the Materials Science and Engineering Laboratory. The objective of the project is to "determine and analyze the mechanical and metallurgical properties and quality of steel, weldments, and connections from steel recovered from WTC 1, 2, and 7."The lab tour/presentation will take place in the Mechanical Properties Lab, where many specimens of steels from structural components of the WTC towers are stored and evaluated.

Tour 2. Nanoscale Elastic-Property Imaging - Donna Hurley

We are developing AFM-based metrology for rapid, nondestructive measurement of mechanical properties with true nanoscale spatial resolution. Our hybrid acoustic/AFM approach involves the vibrational resonance of an AFM cantilever when its tip is in contact with a sample. We have demonstrated how this approach can be used to determine the local indentation modulus of an unknown material or thin film. The AFM's scanning capabilities mean that we can also obtain in-situ images of relative elastic stiffness with nanoscale spatial resolution. Current research is aimed at combining the quantitative, single-point methods with qualitative imaging in order to obtain quantitative maps of nanomechanical properties.

Complementary information obtained with scanning electron microscopy provides insight into structure-property relations and helps to interpret the nanoscale contact-mechanics behavior.

\section{Tour 3. Thin Film Mechanical Testing_-David Read}

We perform microtensile testing on thin films of metals, polymers, and ceramics. A typical gage section is $1 \mu \mathrm{m}$ thick by $10 \mu \mathrm{m}$ wide by $200 \mu \mathrm{m}$ long. The specimen is patterned by 
photolithography on bare silicon. The silicon is etched from beneath the specimen, to a depth of at least $60 \mu \mathrm{m}$, using xenon difluoride. This etch frees the gage section and a grip section with a hole for the loading pin. Tests are done under an optical microscope or inside the SEM. Using a three-axis micromanipulator, the loading pin is placed in the $50 \mu \mathrm{m}$ diameter hole in the grip section of the specimen. As the load is applied, the displacement of a calibrated spring is recorded. A calibration factor, obtained using a force pendulum, is used to convert the measured displacements to force values. Our noise level is tens of micronewtons; failure loads for our specimens range upward from around $1 \mathrm{mN}$. Digital images are recorded every few seconds during the test; digital image correlation is used to obtain the engineering strain in the gage section. The gage section of the specimen is imaged over about 800 pixels of the digital images, and the displacements are obtained with a precision of 0.01 to 0.05 pixels, for a strain noise level of about 60 microstrain. Data are presented in the form of engineering stress-strain curves. Visitors to the lab will be able to see the optical testing station, the micromanipulator, the xenon difluoride etching system, and posters showing typical specimens and results.

\section{Tour 4. MEMS for Testing of Biological Systems - Andrew Slifka}

We are developing MEMS-based mechanical test platforms and tools that can be integrated with currently used biological techniques for the evaluation and measurement of cellular response (e.g., gene expression, cell morphology, area of adhesion, etc.). These types of studies are needed because the development of vascular smooth muscle cells in cardiovascular tissue, for example, is dependent on variations in the stress-strain environment that result from the expansion and contraction of the vessel wall. The importance of the environment becomes apparent when one considers that engineered tissues have mechanical properties inferior to those of naturally grown tissues. This is possibly a bulk effect, but is clearly related to processes at the cellular level. Without a quantitative understanding of the mechanics and functionality of the building blocks (cells and fibers), the bulk properties of the tissues cannot be fully understood and modeled. 


\section{NIST Technical Publications}

\section{Periodical}

Journal of Research of the National Institu te of Standards and Technology-Reports NIST research and development in metrology and related fields of physical science, engineering, applied mathematics, statistics, biotechnology, and information technology. Papers cover a broad range of subjects, with major emphasis on measurement methodology and the basic technology underlying standardization. Also included from time to time are survey articles on topics closely related to the Institute's technical and scientific programs. Issucd six times a year.

\section{Nonperiodicals}

Monographs-Major contributions to the technical literature on various subjects related to the Institute's scientific and technical activities.

Handbooks-Recommended codes of engineering and industrial practice (including safety codes) devel- oped in cooperation with interested industries, professional organizations, and regulatory bodies.

Special Publications-Include proceedings of conferences sponsored by NIST, NIST annual reports, and other special publications appropriate to this grouping such as wall charts, pocket cards, and bibliographies.

National Standard Reference Data Series-Provides quantitative data on the physical and chemical properties of materials, compiled from the world's literature and critically evaluated. Developed under a worldwide program coordinated by NIST under the authority of the National Standard Data Act (Public Law 90-396). NOTE: The Journal of Physical and Chemical Reference Data (JPCRD) is published bimonthly for NIST by the American Institute of Physics (AIP). Subscription orders and renewals are available from AIP, P.O. Box 503284, St. Louis, MO 63150-3284.

Building Science Series-Disseminates technical information developed at the Institute on building materials, components, systems, and whole structures. The series presents research results, test methods, and performance criteria related to the structural and environmental functions and the durability and safety characteristics of building elements and systems.

Technical Notes-Studies or reports which are complete in themselves but restrictive in their treatment of a subject. Analogous to monographs but not so comprehensive in scope or definitive in treatment of the subject area. Often serve as a vehicle for final reports of work performed at NIST under the sponsorship of other government agencies.

Voluntary Product Standards-Developed under procedures published by the Department of Commerce in Part 10 , Title 15, of the Code of Federal Regulations. The standards establish nationally recognized requirements for products, and provide all concerned interests with a basis for common understanding of

the characteristics of the products. NIST administers this program in support of the efforts of private-sector standardizing organizations.

Order the following NIST publications-FIPS and NISTIRs-from the National Technical Information Service, Springfield, VA 22161.

Federal Information Processing Standards Publications (FIPS PUB)-Publications in this series collectively constitute the Federal Information Processing Standards Register. The Register serves as the official source of information in the Federal Government regarding standards issued by NIST pursuant to the Federal Property and Administrative Services Act of 1949 as amended. Public Law 89-306 (79 Stat. 1127), and as implemented by Executive Order 11717 (38 FR 12315, dated May 11,1973) and Part 6 of Title 15 CFR (Code of Federal Regulations).

NIST Interagency or Internal Reports (NISTIR)-The series includes interim or final reports on work performed by NIST for outside sponsors (both government and nongovernment). In general, initial distribution is handled by the sponsor: public distribution is handled by sales through the National Technical Information Service. Springfield, VA 22161. in hard copy, electronic media, or microfiche form. NISTIR's may also report results of NIST projects of transitory or limited interest, including those that will be published subsequently in more comprehensive form. 


\section{U.S. Department of Commerce}

National Bureau of Standards and Technology

325 Broadway

Boulder, CO 80305-3328

\section{Official Business}

Penalty for Private Use $\$ 300$ 